\title{
Study the Effect of Chemical Reaction and Magnetic Field on Free Convection Boundary Layer Flow of Heat and Mass Transfer with Variable Prandtl Number
}

\author{
M. S. Alam ${ }^{1}$, M. Ali ${ }^{* 1}$, M. A. Alim ${ }^{2}$ \\ ${ }^{1}$ Department of Mathematics, Chittagong University of Engineering and Technology, \\ Chittagong, Chittagong-4349, Bangladesh \\ ${ }^{2}$ Department of Mathematics, Bangladesh University of Engineering and Technology, \\ Dhaka-1000, Bangladesh
}

Received 11 July 2015, accepted in final revised form 13 December 2015

\begin{abstract}
The present problem is focused on the free convection heat and mass transfer flow of an electrically conducting incompressible viscous fluid about a semi-infinite vertical plate by the effect of chemical reaction and magnetic field under the action of heat absorption and variable Prandtl nnumber. The governing partial differential equations are transformed to ordinary differential equation by applying local similarity transformation. Then the dimensionless ordinary differential equations are solved using shooting iteration technique along with Runge-Kutta integration scheme. The effects of magnetic parameter and chemical reaction parameter with variable Prandtl number on velocity, temperature and concentration profiles are discussed numerically and shown graphically. Therefore, the results of velocity field decreases for increasing values of magnetic parameter and chemical reaction parameter in both air and salt water. The temperature field decreases in the presence of magnetic parameter but increases for chemical reaction parameter in case of air and salt water. Also, the concentration profile is slightly increased for increasing the values of magnetic parameter but significant decreasing effect are observed for reaction parameter. Finally, the numerical values of the shear stress, rate of temperature and rate of concentration are also shown in a tabular form.
\end{abstract}

Keywords: MHD; Heat absorption; Free convection; Chemical reaction.

(C) 2016 JSR Publications. ISSN: 2070-0237 (Print); 2070-0245 (Online). All rights reserved. doi: http://dx.doi.org/10.3329/jsr.v8i1.25599 J. Sci. Res. 8 (1), 41-48 (2016)

\section{Introduction}

Heat and mass transfer analysis with chemical reaction has a great interest to engineers and scientist because of its universal applications in many branches of science and

* Corresponding author: ali.mehidi93@gmail.com 
engineering. In many chemical processes, a chemical reaction occurs between a foreign mass and a fluid in which a plate is moving. These processes have numerous applications such as polymer production, manufacturing of ceramics or glassware and food processing. Besides, the magneto hydrodynamic laminar boundary layer behavior over a stretching surface is a significant type of flow having considerable practical applications in chemical engineering, electrochemistry and polymer processing. This problem has also an important bearing on metallurgy where magneto hydrodynamic (MHD) techniques have recently been used. In this regard, recently, Ali and Alam [1] has investigated the heat and mass transfer on steady MHD free convection flow along an inclined stretching sheet with suction and heat generation, Rahman et al. [2] studied the effect of joule heating on MHDs mixed convection around a heat conducting horizontal circular cylinder in a rectangular lid-driven cavity, Kuiry and Bhadur [3] has analyzed the effect of inclined magnetic field of viscous fluid between two parallel porous plates with heat transfer, Ahmmed and Sarker [4] studied the MHD natural convection flow of fluid from a vertical flat plate considering temperature dependent viscosity, Reddy [5] has studied the influence of MHD and thermal radiation boundary layer flow of a nanofluid past a stretching sheet, Kumar and Sing [6] have also studied the mathematical modeling of Soret and hall effects on oscillatory MHD free convective flow of radiating fluid in a rotating vertical porous channel filled with porous medium, Sing [7] studied the heat and mass transfer in MHD boundary layer flow past an inclined plate with viscous dissipation in porous medium, Abel and Mahesh [8] presented an analytical and numerical solution for heat transfer in a steady laminar flow of an incompressible viscoelastic fluid over a stretching sheet with power-law surface temperature, including the effects of variable thermal conductivity and non-uniform heat source and radiation, Iskak et al. [9] discussed heat transfer analysis for mixed convection flow through a vertical stretching sheet, Ali et al. [10] have analyzed the MHD viscos flow of heat transfer over a permeable shrinking sheet with surface heat flux, Rajesh [11] has studied the MHD effects on free convection and mass transform flow through a porous medium with variable temperature and Alam et al. [12] discussed the steady MHD boundary layer free convective heat and mass transfer flow over an inclined porous plate with variable suction and Soret effect in presence of hall current, Ferdows et al. [13] discussed the boundary layer flow of a nanofluid through a porous medium due to an Exponentially Stretching sheet in presence of magnetic field, Khan et al.[14] have analyzed the effects of magnetic field on radiative flow of a nanofluid past a stretching sheet. Since the study of heat and mass transfer is important in many cases, in the present paper we studied the free convection heat and mass transfer flow over a semi-infinite vertical plate with heat absorption, chemical reaction and magnetic field in presence of variable Prandtl number. The boundary layer equations are transformed by a similarity transformation into a system of coupled non-linear ordinary differential equations and which are solved numerically by shooting iteration technique along with Runge- Kutta sixth order method. 


\section{Mathematical Formulation and Governing Equations of the Problem}

Let us consider a two dimensional steady laminar MHD viscous incompressible electrically conducting fluid along a vertical plate. A magnetic field of strength $B_{0}$ has been applied to the normal of the direction of the flow. The uniform plate temperature $T w\left(>T_{\infty}\right)$, where $T_{\infty}$ is the temperature of the fluid far away from the plate. Let $u$ and $v$ be the velocity components along the $x$ and $y$ axes respectively in the boundary layer region. Under the above assumptions and usual boundary layer approximation, the dimensional governing equations of continuity, momentum, concentration and energy under the influence of externally imposed magnetic field are [2]:

Equation of continuity:

$$
\frac{\partial u}{\partial x}+\frac{\partial v}{\partial y}=0
$$

Momentum equation:

$$
u \frac{\partial u}{\partial x}+v \frac{\partial u}{\partial y}=v \frac{\partial^{2} u}{\partial y^{2}}+g \beta\left(T-T_{\infty}\right)+g \beta^{*}\left(C-C_{\infty}\right)-\frac{\sigma B_{0}^{2} u}{\rho}
$$

Energy Equation:

$$
u \frac{\partial T}{\partial x}+v \frac{\partial T}{\partial y}=\frac{\kappa}{\rho c_{\mathrm{p}}} \frac{\partial^{2} T}{\partial y^{2}}+Q^{*}\left(\mathrm{C}-\mathrm{C}_{\infty}\right)
$$

Concentration Equation:

$$
u \frac{\partial C}{\partial x}+v \frac{\partial C}{\partial y}=D_{m} \frac{\partial^{2} C}{\partial y^{2}}-\lambda^{*}\left(C-C_{\infty}\right)
$$

Boundary conditions are:

$u=U_{0} x, v=0, T=T_{w}, C=C_{w}$ at $y=0$,

$u=0, T=T_{\infty}, C=C_{\infty}$ as $y \rightarrow \infty$

To convert the governing equations into a set of similarity equations, we introduce the following similarity transformation:

$\eta=y \sqrt{\frac{U_{0}}{v x}}, \psi=\sqrt{U_{0} v x} f(\eta), \theta(\eta)=\frac{T-T_{\infty}}{T_{w}-T_{\infty}}, \varphi(\eta)=\frac{C-C_{\infty}}{C_{w}-C_{\infty}}, u=\frac{\partial \psi}{\partial y}, v=-\frac{\partial \psi}{\partial x}$

By using the above transformations, the equations (2)-(4) are reduced to nondimensional, nonlinear and coupled ordinary differential equations: 


$$
\begin{aligned}
& f^{\prime \prime \prime}+\frac{1}{2} f f^{\prime \prime}-M f^{\prime}+G r \theta+G m \phi=0 \\
& \theta^{\prime \prime}+\frac{1}{2} \operatorname{Pr}\left(f \theta^{\prime}-f^{\prime} \theta\right)+\operatorname{Pr} R_{a} \varphi=0 \\
& \varphi^{\prime \prime}+\frac{1}{2} S c\left(f \varphi^{\prime}-f^{\prime} \varphi\right)-S c C_{r} \varphi=0
\end{aligned}
$$

The transform boundary conditions:

$$
f=0, f^{\prime}=1, \theta=\varphi=1 \text { at } \eta=0, f^{\prime}=\theta=\varphi=0 \text { as } \eta \rightarrow \infty
$$

Where $f^{\prime}, \theta$ and $\varphi$ are the dimensionless velocity, temperature and concentration profiles respectively, $\eta$ is the similarity variable, the prime denotes differentiation with respect to $\eta$.

Also the non-dimensional parameters are

$$
G r=\frac{g \beta q x}{U_{0}^{2} \kappa} \sqrt{\frac{\nu x}{U_{0}}}, G m=\frac{g \beta^{*}\left(C_{w}-C\right) x}{U_{0}^{2}} \sqrt{\frac{\nu x}{U_{0}}}, M=\frac{\sigma B_{0} x}{\rho U_{0}}, \operatorname{Pr}=\frac{v}{\alpha}, Q=\frac{\kappa m Q^{*} x}{U_{0} D_{m}}, C=\frac{\lambda^{*} x}{U_{0}}, S c=\frac{v}{D_{m}}
$$

Which indicate the Grashof number, modified Grashof number, magnetic parameter, Prandtl number, heat absorption parameter, chemical reaction parameter and Schmidt number respectively.

\section{Results and Discussion}

Numerical calculation for distribution of the velocity, temperature and concentration profiles across the boundary layer are displayed in Figs. 1 - 12 for different values of magnetic parameter $M$, and chemical reaction parameter $C$ with variable Prandtl number and fixed values of Schmidt number $S c$, heat absorption parameter $R$, Grashof number $G r$ and modified Grashof number $G m$. The values of Prandtl number $P r$ are chosen for 1.00 and 0.71 which correspond to salt water and air and also Schmidt number are taken as 0.66 which correspond to water vapor.

The effects of various parameters on velocity profile are shown in Figs. 1- 4. From Figs. 1 and 2 it is observed that the velocity decreases with an increase in the $M$. The magnetic parameter is found to retard the velocity at all points of the flow field. It is because that the application of transverse magnetic field will result in a resistive type force (Lorentz force) similar to drag force which tends to resist the fluid flow and thus reducing its velocity. Similar result has been found both in the cases of air and salt water for increasing values of reaction parameter which are shown in Figs . 3 and 4.

The effect of various parameters on temperature profile are shown in Figs.5-8. From these Figures we see that, the temperature profile is starting at the initial point of 
the plate surface and then increasing until it reaches to a maximum point and then decreases far away from the plate satisfying the boundary condition. Figs. 5 and 6 show the temperature distribution for different values of magnetic parameter $M$ and observed that the thermal boundary layer decreases as $M$ increases adjacent to the surface of the plate and the effect is not significant far away from the plate but reverse result arises for the increasing values of chemical reaction parameter both of air and salt water which are shown in Figs.7 and 8.

Figs. 9-12 show the concentration profiles obtained by the numerical simulation for various values of entering non-dimensional parameters. From Figs. 9 and 10 the negligible increasing effect is observed on concentration profiles for increasing values of $M$ in case of air and salt water. Again the significant decreasing effect are observed for increasing values of chemical reaction parameter in both of air and salt water which are shown in Figs.11 and 12.

Again, from Table 1 it is observed that the skin friction is decreased for magnetic parameter and reaction parameter as a result the momentum boundary layer is decreased. The rate of heat transfer is increased for magnetic parameter but reverse trend arises for reaction parameter in both of air and salt water. Again, the rate of concentration is decreased for magnetic parameter but reverse trend arises for chemical reaction parameter in both of air and salt water as a result the concentration boundary layer is increased for $M$ and decreased for $C$.

Table 1. The skin friction $f^{\prime}(0)$, rate of heat transfer $-\theta^{\prime}(0)$ and rate of concentration $-\varphi^{\prime}(0)$ for

\begin{tabular}{cccccccccc}
\hline $\mathrm{M}$ & $\mathrm{C}$ & \multicolumn{2}{c}{$\operatorname{Pr}$} & \multicolumn{2}{c}{$\mathrm{f}^{\prime}(0)$} & \multicolumn{2}{c}{$\varphi^{\prime}(0)$} & \multicolumn{2}{c}{$-\theta^{\prime}(0)$} \\
\hline 2.0 & 0.5 & 0.71 & 1.0 & -1.1241 & -1.1713 & 0.4231 & 0.4215 & 1.2913 & 2.0778 \\
2.5 & 0.5 & 0.71 & 1.0 & -1.2382 & -1.2803 & 0.4146 & 0.4132 & 1.2479 & 1.9975 \\
3.0 & 0.5 & 0.71 & 1.0 & -1.3447 & -1.3825 & 0.4042 & 0.4023 & 1.2019 & 1.9119 \\
1.0 & 0.5 & 0.71 & 1.0 & -1.0521 & -1.0754 & 0.4412 & 0.4435 & 1.2356 & 1.7965 \\
1.0 & 1.5 & 0.71 & 1.0 & -1.1274 & -1.1754 & 0.5975 & 0.5969 & 1.4363 & 2.2958 \\
0.5 & 2.5 & 0.71 & 1.0 & -1.1309 & -1.1796 & 0.7443 & 0.7439 & 1.5911 & 2.5297 \\
\hline
\end{tabular}

different values of M, C and Pr with Sc 0.66 by considering hot plate.
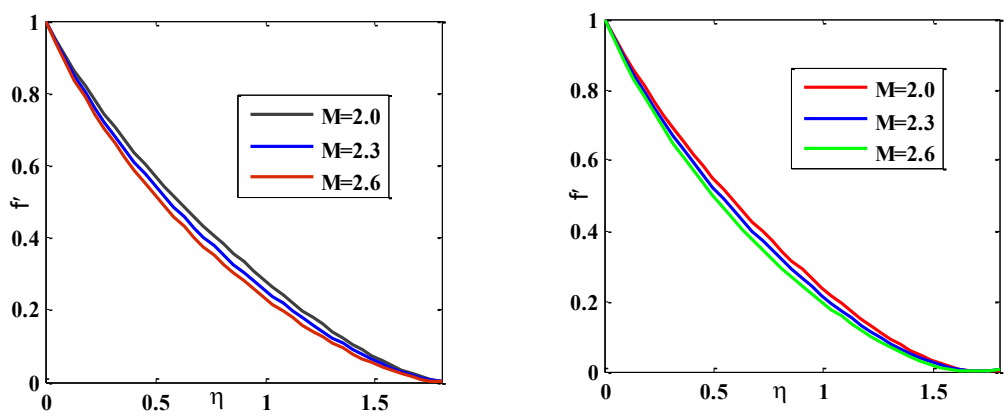

Fig. 1. Velocity profile for $\mathrm{M}$ in case of air. Fig. 2. Velocity profile for $\mathrm{M}$ in case of water. 


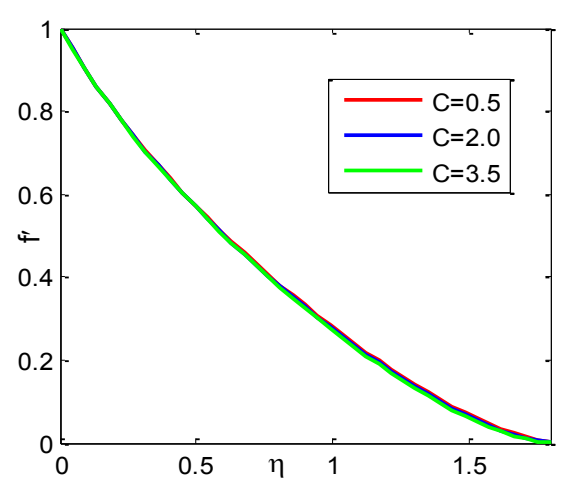

Fig. 3. Velocity profile for $\mathrm{C}$ in case of air.

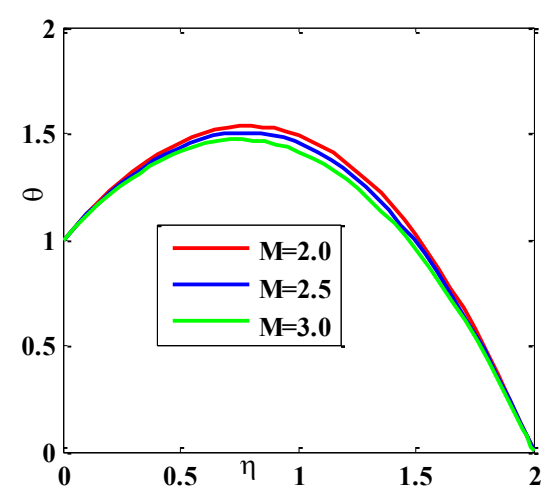

Fig. 5. Temperature profile for $M$ in case of air.

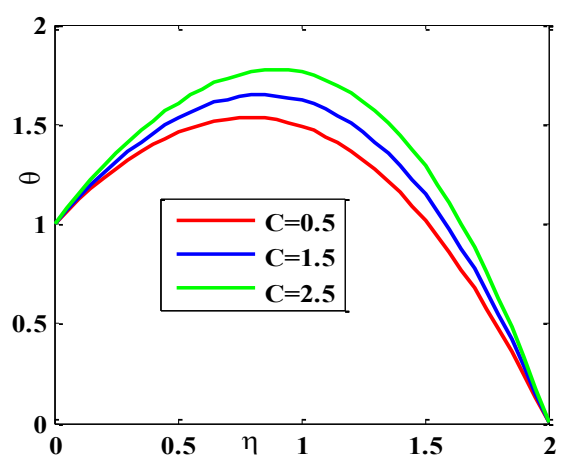

Fig. 7. Temperature profile for $\mathrm{C}$ in case of air.

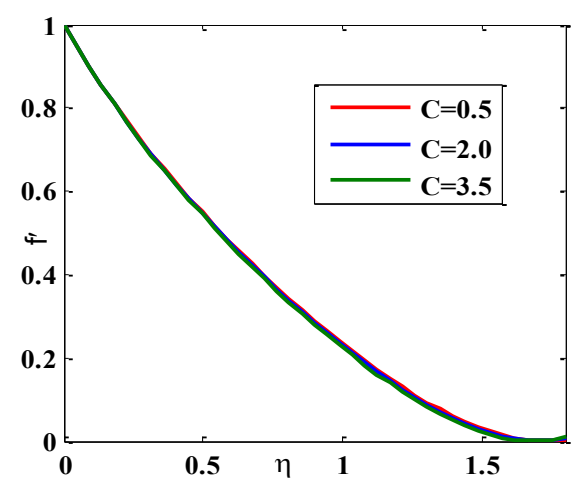

Fig. 4. Velocity profile for $\mathrm{C}$ in case of water.

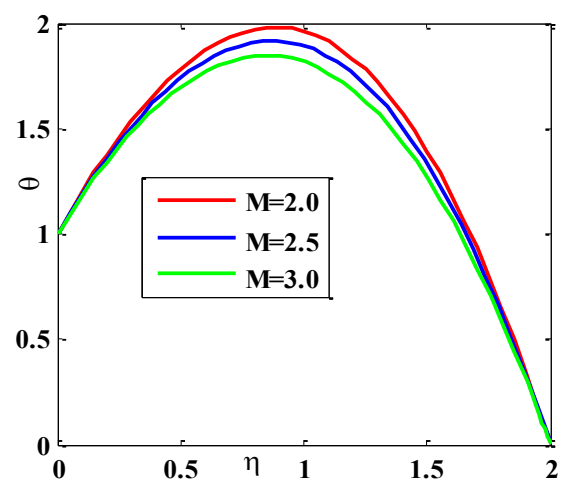

Fig. 6. Temperature profile for $\mathrm{M}$ in case of water.

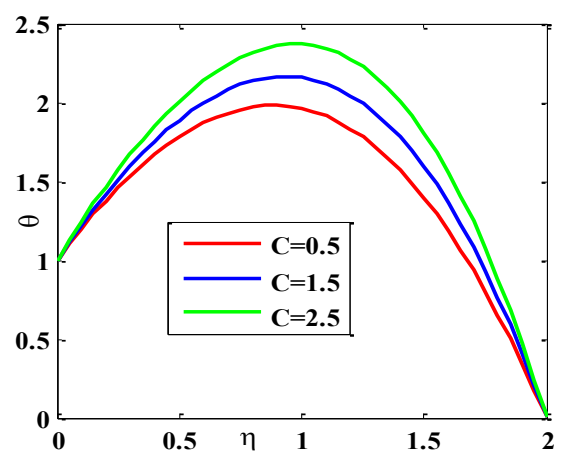

Fig. 8. Temperature profile for $\mathrm{C}$ in case of water. 


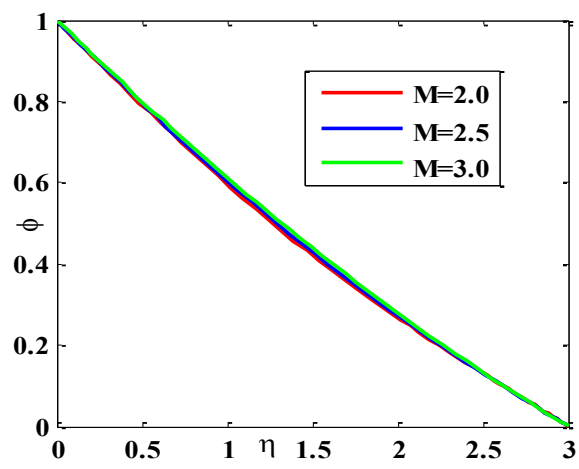

Fig. 9. Concentration profile for $\mathrm{M}$ in case of air.

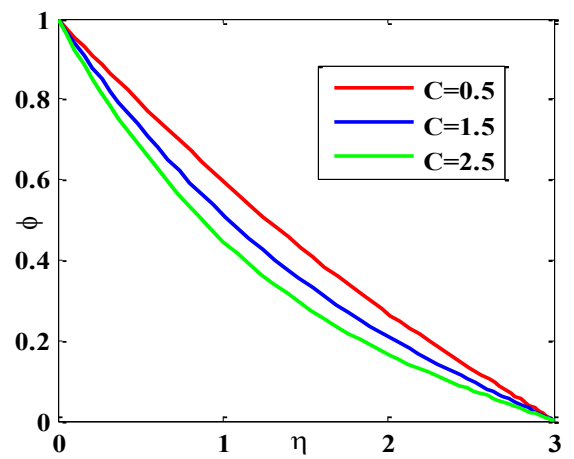

Fig. 11. Concentration profile for $\mathrm{M}$ in case of air.

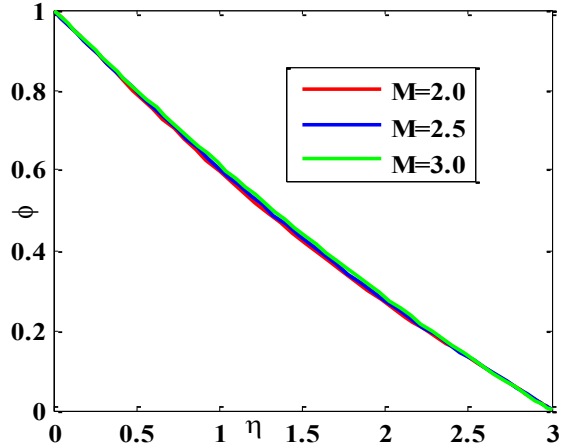

Fig. 10. Concentration profile for $\mathrm{M}$ in case of water.

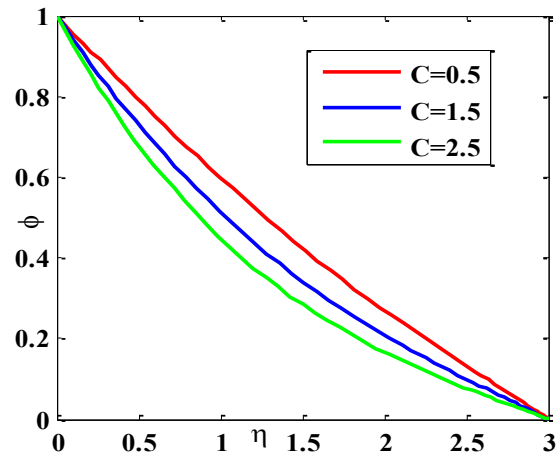

Fig. 12. Concentration profile for $\mathrm{M}$ in case of water.

\section{Conclusion}

The results are presented to display the flow characteristic like velocities, temperature and concentration. Followings are the conclusions made from above analyses:

- The magnitude of velocity decreases in both the air and water with increasing magnetic parameter causing of Lorentz force. Also the negligible decreasing effect are observed for the influence of reaction parameter on the velocity profile.

- The temperature decreases in both air and water with an increase in magnetic parameter but noticeable increasing effect for chemical reaction parameter.

- The concentration profile is slightly increase for magnetic parameter in both air and water but decreased for chemical reaction parameter. 


\section{Nomenclature}

\begin{tabular}{|c|c|c|c|}
\hline $\begin{array}{l}\text { MHD } \\
c_{\eta}\end{array}$ & $\begin{array}{l}\text { Magneto hydrodynamics } \\
\text { Specific heat of with constant }\end{array}$ & & $\begin{array}{l}\text { Velocity component in } \mathrm{x}- \\
\text { direction }\end{array}$ \\
\hline p & $\begin{array}{l}\text { pressure } \\
\text { Gravitational acceleration }\end{array}$ & $\mathrm{v}$ & Velocity component in $y-$ \\
\hline $\begin{array}{l}g \\
f^{\prime}\end{array}$ & $\begin{array}{l}\text { Gravitational acceleration } \\
\text { Velocity Profile }\end{array}$ & $\mathrm{T}$ & $\begin{array}{l}\text { direction } \\
\text { Temperature }\end{array}$ \\
\hline$v$ & Kinematic viscosity & $\mathrm{D}_{\mathrm{m}}$ & Thermal molecular diffusivity \\
\hline$\eta$ & Similarity variable & $\mathrm{k}$ & Thermal conductivity \\
\hline$\alpha$ & $\begin{array}{l}\text { Thermal } \\
\alpha=\frac{\mathrm{k}}{\rho \mathrm{c}_{\mathrm{p}}}\end{array}$ & $\begin{array}{l}C_{\infty} \\
B_{0}\end{array}$ & $\begin{array}{l}\text { Concentration of the fluid } \\
\text { outside the boundary layer } \\
\text { Constant magnetic field } \\
\text { intensity }\end{array}$ \\
\hline$\beta$ & Expansion & $\mathrm{U}_{0}$ & $\begin{array}{l}\text { Constant velocity } \\
\text { Heat absorption constant }\end{array}$ \\
\hline$\beta^{*}$ & Coefficient of expansion with & $\lambda^{*}$ & Reaction rate constant \\
\hline$\rho$ & $\begin{array}{l}\text { concentration } \\
\text { Density }\end{array}$ & $T_{w}$ & Temperature at the Plate \\
\hline$\sigma$ & Fluid electrical conductivity & $T_{\infty}$ & $\begin{array}{l}\text { Temperature of the fluid } \\
\text { outside the boundary layer }\end{array}$ \\
\hline
\end{tabular}

\section{References}

1. M. Ali and M. S. Alam, J. Sci. Res. 6, 457 (2014). http://dx.doi.org/10.3329/jsr.v6i3.16903

2. M. M. Rahman, M. A. Alim, and M. K. Chowdhury, J. Sci. Res. 1, 461 (2009). http://dx.doi.org/10.3329/jsr.vli3.2597

3. D. R. Kuiry and S. Bahadur, J. Sci. Res. 7, 21 (2015). http://dx.doi.org/10.3329/jsr.v7i3.22574

4. S. F. Ahmmed and M. S. A. Sarker, J. Sci. Res. 2, 453 (2010). http://dx.doi.org/10.3329/jsr.v2i3.4776

5. M. G. Reddy, J. Sci. Res. 6, 257 (2014). http://dx.doi.org/10.3329/jsr.v6i2.17233

6. R. Kumar and K. D. Singh, Int. J. Appl. Math. Mech. 8, 49 (2012).

7. P. K Sing, J. Sci. Engg. Res. 3, 1 (2012).

8. M. S. Abel and N. Mahesha, Appl. Math. Modl. 32, 1965 (2008). http://dx.doi.org/10.1016/j.apm.2007.06.038

9. A. Ishak and R. Nazar, Int. Pop, H. M. Trans. 44, 921 (2008). http://dx.doi.org/10.1007/s00231-007-0322-z

10. F. M. Ali, R. Nazar, and N.M. Arifin, WSEAS Trans. Math. 9, 365 (2010).

11. V. Rajesh, Int. J. Appl. Math. Mech. 6, 1 (2010).

12. M. S. Alam, M. Ali, M. A. Alim, and A. Saha, Bangladesh J. Sci. Ind. Res. 49, 155 (2014). http://dx.doi.org/10.3329/bjsir.v49i3.22129

13. M. Ferdows, M. S. Khan, M. M. Alam, and S. Sun, Math. Prob. Eng. 3, 2551 (2012).

14. M. S. Khan, M. M. Alam and M. Ferdows, Pro. Engg. Elsevier, 56, 316 (2013). http://dx.doi.org/10.1016/j.proeng.2013.03.125 